\title{
Trastornos temporomandibulares: Perfil clínico, comorbilidad, asociaciones etiológicas y orientaciones terapéuticas
}

\section{Temporomandibular disorders: clinical profile, comorbility, etiological associations and therapeutical guidelines} Meeder Bella $\mathrm{W}^{*}$, Weiss Vega $\mathrm{F}^{* *}$, Maulén Yañez $\mathrm{M}^{* * *}$, Lira Alegría $\mathrm{D}^{* * *}$, Padilla
Ladrón de Guevara $\mathrm{R}^{* *}$, Hormazábal Navarrete $\mathrm{F}^{* * * *}$, Guerrero Marholz L****

\section{RESUMEN}

Objetivo: El propósito de la presente investigación fue dilucidar factores etiológicos y determinar la comorbilidad en enfermos con trastornos temporomandibulares (TTM) y dolor orofacial (DOF), con el objetivo de definir orientaciones terapéuticas.

Materiales y métodos: Se realizó un estudio clínico-anamnésico entre abril de 2004 y abril de 2008, en pacientes que concurrieron en forma espontánea a la clínica universitaria de la especialidad.

Resultados: Se examinaron 263 pacientes, de los cuales 80,22\% fueron mujeres. El motivo de consulta más prevalente fue dolor cráneo-cérvicofacial (84\%). El 49,43\% de los pacientes no reconoció la causa de inicio del problema, siendo "estrés/depresión" (24,71\%) la más referida. Los TTM representaron una condición eminentemente crónica: el 34,55\% de los pacientes relataron un tiempo de evolución entre 1-5 años, solo un $17,58 \%$ tuvo una evolución menor de 6 meses. La frecuencia relativa de diagnósticos, no excluyentes, fue: dolor muscular local, 81,37\%; capsulitis/sinovitis, 74,52\%; y desórdenes psicosociales, 61,98\%. Se observó alta comorbilidad con cefaleas, 55,89\%; alteraciones psicosociales, 52,47\%; disturbios del sueño, 39,54\%, y alteraciones cervicales, $28,9 \%$

Discusión: Las características sociodemográficas y clínicas de los pacientes evaluados, se corresponden con los reportes de la literatura actual. Se encontraron asociaciones significativas entre: bruxismo y dolor muscular local $(p=0,014)$; bruxismo y capsulitis/sinovitis $(p=0,025)$, y depresión severa/moderada con dolor muscular local $(p=0,006)$. Se concluye que para el tratamiento de pacientes con TTM es necesaria la autorregulación física, el control de parafunciones y la colaboración interdisciplinaria.

Palabras clave: Trastornos temporomandibulares, dolor orofacial, dolor crónico, comorbilidad, trastornos psicosociales.

\section{SUMMARY}

Purpose: The aim of this study was to elucidate the etiologic factors and assess comorbidity in patients with temporomandibular disorders (TMD) and orofacial pain (OFP), to define therapeutic guidelines.

* Cirujano dentista. Profesor titular. Jefe de la Cátedra de Fisiología Oral y Trastornos Temporomandibulares. Director del Programa de Especialización en Trastornos Temporomandibulares y Dolor Orofacial. Facultad de Odontología. Universidad de Valparaíso; y Policlínico Armada de Chile.

** Cirujano dentista. Profesor auxiliar. Cátedra de Fisiología Oral y Trastornos Temporomandibulares. Facultad de Odontología. Universidad de Valparaíso; y Servicio de Salud Valparaíso. San Antonio.

*** Cirujano dentista. Profesor adjunto, Cátedra de Fisiología Oral y Trastornos Temporomandibulares, Facultad de Odontología. Universidad de Valparaíso; y Servicio de Salud Viña del Mar. Quillota.

**** Cirujano dentista. Residente Programa de Especialización en Trastornos Temporomandibulares y Dolor Orofacial. Facultad de Odontología. Universidad de Valparaíso; y Servicio de Salud Araucanía Sur. 
Materials and methods: A clinical-anamnestic study was done between April 2004 and April 2008 in patients who spontaneously attended the university clinic of the specialty.

Results: 263 patients were examined, of whom $80.22 \%$ were women. The most common complaint was cranio facial pain (84\%). A $49.43 \%$ of the patients did not recognize the cause of onset of the problem. "Stress / depression" (24.71\%) was the most mentioned. TMD eminently represented a chronic condition: the $34.55 \%$ of patients reported a duration time between $1-5$ years, only $17.58 \%$ had the pathology less than 6 months. The relative frequency of diagnoses, non exclusive, was local muscle pain, $81.37 \%$; capsulitis / synovitis, $74.52 \%$, and psychosocial disorders, 61.98\%. High comorbidity of TMD was observed with headache, 55.89\%; psychosocial disorders, 52.47\%; sleep disturbances, 39.54\%; and cervical abnormalities, $28.9 \%$.

Discussion: The sociodemographic and clinical features of patients that were evaluated match reports of the current literature. We found significant associations between bruxism and local muscle pain ( $p=0,014)$; bruxism and capsulitis / synovitis $(p=0,025)$, and depression / moderate and local muscle pain $(p=0,006)$. We conclude that physical self regulation, parafunction control and interdisciplinary collaboration are needed as treatment for patients with TMD.

Key words: TMD, orofacial pain, chronic pain, comorbidity, psychosocial disorders.

Fecha de recepción: 29 de abril 2009.

Aceptado para publicación: 7 de julio 2009.

Meeder Bella W, Weiss Vega F, Maulén Yañez M, Lira Alegría D, Padilla Ladrón de Guevara R, Hormazábal Navarrete F, Guerrero Marholz L. Trastornos temporomandibulares: Perfil clínico, comorbilidad, asociaciones etiológicas y orientaciones terapéuticas. Av. Odontoestomatol 2010; 26 (4): 209-216.

\section{INTRODUCCIÓN}

Los trastornos temporomandibulares (TTM) se definen como un conjunto de diversos problemas clínicos que pueden involucrar la musculatura masticatoria, las articulaciones temporomandibulares o ambas (1). En general los estudios de los TTM se han caracterizado por: la aplicación de diferentes metodologías de examen y recolección de datos; la evaluación de signos y síntomas por sobre categorías diagnósticas; el empleo de distintas clasificaciones diagnósticas; y la utilización de categorías generales. Esto ha impedido establecer diagnósticos específicos y por niveles. También se han establecido criterios arbitrarios y escalas de severidad en variables continuas o ponderación relativa de factores causales no validadas adecuadamente (2-6).

Por otra parte, la etiología de los TTM fue valorada durante muchos años, desde una perspectiva estrictamente somática, basada en la premisa de que todo dolor se origina en un daño tisular evidencia- ble. Sin embargo, es necesario contemplar ciertos elementos neuronales, periféricos y/o centrales, que muchas veces se encuentran presentes en los cuadros de dolor crónico, en los cuales la condición de injuria hística periférica no siempre se puede determinar con exactitud, o simplemente, ya no se encuentra presente. En la evaluación de los enfermos con TTM y DOF se deben considerar además, aspectos psicosociales, que muchas veces son agravantes en la génesis y el desarrollo de estas patologías.

En el ámbito odontológico, las diversas "filosofías oclusales" que se desarrollaron al alero de las disciplinas rehabilitadoras intentaron circunscribir la problemática diagnóstica y terapéutica de los TTM exclusivamente al componente dento-oclusal.

El propósito del presente estudio fue determinar condiciones sociodemográficas, anamnésicas y clínicas relevantes y definir asociaciones de comorbilidad y causalidad en pacientes con TTM y DOF, con el objetivo de definir orientaciones terapéuticas. 


\section{MATERIALES Y MÉTODOS}

Se realizó un estudio clínico entre abril del 2004 y abril del 2008. Los pacientes fueron recepcionados por un profesor de la especialidad, quien luego de la entrevista preliminar, concluía si el paciente sufría efectivamente un TTM. Posteriormente se realizaba la evaluación por los residentes de la especialidad, instruidos y calibrados, tanto para el examen sistemático del paciente, como para la aplicación de las encuestas, por un período de tres meses. Los datos fueron recopilados aplicando un método de examen sistemático desarrollado en la Clínica de Trastornos Temporomandibulares y Dolor Orofacial de la Facultad de Odontología de la Universidad de Valparaíso. La Ficha incorporó un total de 212 variables.

Para la evaluación clínica se utilizaron los métodos de examen clínico y las categorías y subcategorías diagnósticas descritas por la Academia Americana de Dolor Orofacial (7), con algunas complementaciones realizadas por la Cátedra de Fisiología Oral y Trastornos Temporomandibulares.

La condición psicosocial fue evaluada a través del "SCL-90" (Symtom Checklist-90-Revised) adaptado (8) y el "Índice de Calidad de Sueño de Pittsburg" (9).

Los resultados de este examen y la evaluación psicosocial fueron traspasados a la base de datos de la especialidad. Los datos se sometieron a un análisis descriptivo con tablas de frecuencia absoluta y relativa (\%) en conjunto con gráficas, con el propósito de resumir la información. El análisis de asociación se realizó a través de tablas de contingencia para las variables de mayor interés.

Como plataforma de gestión de datos se utilizó Excel 2007 y los adds in XLSTAT 2008 ver.4.01 y la aplicación DDXL 2008 ver. 1.1. Las asociaciones de variables fueron realizadas con el software estadístico Minitab 14.0.

Las condiciones estudiadas fueron divididas en:

- Antecedentes sociodemográficos, edad y género.

- Aspectos anamnésicos: motivo de consulta, frecuencia relativa de síntomas, tiempo de evolución, forma de inicio del problema, presencia de hábitos parafuncionales y comorbilidad.
- Aspectos del examen físico: laxitud ligamentaria sistémica (10), movimientos cervicales, presencia de ruidos articulares, dolor a la palpación muscular y articular, y alteraciones dento-oclusales (11).

- Categorías diagnósticas: diagnóstico físico y psicosocial.

\section{RESULTADOS}

El $80,22 \%$ de los pacientes fueron mujeres y un $19,77 \%$ hombres $(n=263)$. El rango de edad osciló entre los 8 y 83 años con un promedio de edad de $30,7 \pm 15,7$ años. El rango etario más observado fue entre los 19 y 29 años (38,76\%).

Según el motivo de consulta, la mayor distribución fue por dolor cráneo-cérvicofacial, (31,94\%); apretamiento y/o rechinamiento dentario, $(21,67 \%)$; y ruido articular, (16,35\%). La frecuencia relativa de síntomas se presenta en la tabla 1. Las variables no son excluyentes entre sí.

De acuerdo al tiempo de evolución del problema, el $32,9 \%$ de los pacientes presentó una evolución de entre 1 y 5 años; un 23,9\% mayor a 5 años; un 23,5\% entre 6 meses y 1 año; y un 19,6\% hasta 6 meses.

Respecto de la forma de inicio, el grupo mayoritario $(49,4 \%)$, no identificó la causa de su problema. Dentro de las causas relatadas, la más frecuente $(24,7 \%)$ fue la variable "estrés/depresión"; "bostezando", 7,9\%; y "masticando", 6,1\%.

La frecuencia relativa de malos hábitos y parafunciones, no excluyentes, fue mayor para "apretamiento y rechinamiento", con un $84,3 \%$ y un $62,5 \%$ respectivamente, seguido de "morder labios y mejillas" con un $42,2 \%$. Onicofagia y bricomanía se observaron en el $28 \%$ de los pacientes, mientras que posturas cervicales anormales mantenidas fueron reportadas por un $27,75 \%$ de los sujetos. En cuanto a la comorbilidad, un 55,9\% de los pacientes reportó cefaleas; $17 \%$ afecciones de oído y alergias; y $14,8 \%$ sinusitis.

La aplicación del test de hiperlaxitud sistémica de Beighton arrojó que sólo un 26,23\% $(n=69)$ de los 


\section{TABLA 1.- FRECUENCIA RELATIVA DE SÍNTOMAS}

\begin{tabular}{|l|c|c|c|c|c|c|}
\hline Síntomas (n=263) & $\mathbf{n}$ & Frec. relat. & Hombres & $\%$ & Mujeres & $\%$ \\
\hline Ruido articular & 209 & 79,47 & 35 & 16,75 & 174 & 83,25 \\
Dolor oído, sien o mejilla & 181 & 68,82 & 31 & 17,13 & 150 & 82,87 \\
Dolor cabeza, cuello o mandíbula & 177 & 67,3 & 30 & 16,95 & 147 & 83,05 \\
Rigidez mandibular & 169 & 64,26 & 28 & 16,57 & 141 & 83,43 \\
Dolor al abrir la boca & 147 & 55,89 & 21 & 14,28 & 126 & 85,72 \\
Dolor al masticar o hablar & 145 & 55,13 & 22 & 15,17 & 123 & 84,83 \\
Bloqueo mandibular & 105 & 39,92 & 15 & 14,28 & 90 & 85,72 \\
Desgaste dentario & 106 & 40,3 & 27 & 25,46 & 79 & 74,54 \\
Cambios dento-oclusales & 85 & 32,32 & 18 & 21,18 & 67 & 78,82 \\
Tto. por dolor craneofacial inexplicable & 54 & 20,53 & 8 & 14,81 & 46 & 85,19 \\
\hline
\end{tabular}

pacientes presentaban registros iguales o superiores a 3 puntos, requisito necesario para diagnosticar hiperlaxitud.

La presencia de dolor en los movimientos cervicales (flexión-extensión, rotación e inclinación) se observó en un $45,62 \%$ de los pacientes. El 49,7\% de los pacientes manifestó dolor a la palpación muscular cervical, siendo el trapecio superior el músculo más afectado.

El 74,9\% de los pacientes presentó dolor a la palpación de la musculatura masticatoria, siendo el masetero superficial el más afectado con un $71,1 \%$.

La palpación de la ATM reveló dolor en el polo lateral en un $57,03 \%(n=150)$, y un $62,74 \%(n=165)$ en la zona retrodiscal. Un $46,76 \%$ de los pacientes presentó ruidos articulares.

La media de la apertura máxima voluntaria (sin dolor) fue $38,35 \pm 10,6 \mathrm{~mm}$; un 47,72\% $(n=126)$ de los pacientes con registros menores de $40 \mathrm{~mm}$. La tabla 2 muestra la distribución de las características dento-oclusales. Las variables no son excluyentes entre sí.

Los diagnósticos por niveles más prevalentes se resumen en la tabla 3. Las variables no son excluyentes entre sí. Los hallazgos encontrados para el com- ponente psicosocial se resumen en la tabla 4. Las variables depresión y somatización no son excluyentes entre sí.

\section{TABLA 2.- FRECUENCIA DE CARACTERÍSTICAS DENTO-OCLUSALES}

\begin{tabular}{|l|r|r|}
\hline $\begin{array}{l}\text { Alteraciones dento-oclusales } \\
\text { (n=263) }\end{array}$ & $\mathbf{n}$ & Frec. relat. \\
\hline $\begin{array}{l}\text { Pérdida de más de 5 dientes } \\
\text { posteriores }\end{array}$ & 38 & 14,45 \\
\hline $\begin{array}{l}\text { Deslizamiento mayor a 2 mm } \\
\text { de RC a MIC }\end{array}$ & 22 & 8,37 \\
\hline Mordida cruzada & 33 & 12,55 \\
\hline Mordida abierta lateral & 3 & 1,14 \\
\hline Mordida abierta anterior & 16 & 6,08 \\
\hline Mordida invertida anterior & 9 & 3,42 \\
\hline $\begin{array}{l}\text { Retracción gingival (al menos } \\
1 \text { grupo) }\end{array}$ & 60 & 22,81 \\
\hline $\begin{array}{l}\text { Abrasiones cervicales (al menos } \\
1 \text { grupo) }\end{array}$ & 40 & 15,21 \\
\hline $\begin{array}{l}\text { Desgaste o atriciones (al menos } \\
1 \text { grupo) }\end{array}$ & 94 & 35,74 \\
\hline
\end{tabular}




\begin{tabular}{|l|r|r|c|c|c|c|}
\hline \multicolumn{5}{|c|}{ TABLA 3.- FRECUENCIA DE CATEGORÍAS DIAGNÓSTICAS } \\
\hline Diagnósticos (n=263) & $\mathbf{n}$ & Frec. relat. & Hombres & $\%$ & Mujeres & $\%$ \\
\hline Ruido articular & 209 & 79,47 & 35 & 16,75 & 174 & 83,25 \\
Contracción protectora & 45 & 17,11 & 7 & 15,6 & 38 & 84,4 \\
Dolor muscular local & 214 & 81,37 & 38 & 17,8 & 176 & 82,2 \\
Luxación espontánea & 2 & 0,76 & 1 & 50 & 1 & 50 \\
Subluxación & 90 & 34,22 & 14 & 15,6 & 76 & 84,4 \\
Desplazamiento discal con reducción & 144 & 54,75 & 26 & 18,1 & 118 & 81,9 \\
Osteoartritis & 17 & 6,46 & 3 & 17,6 & 14 & 82,4 \\
Retrodiscitis & 146 & 55,51 & 24 & 16,4 & 112 & 76,7 \\
Capsulitis/sinovitis & 196 & 74,52 & 36 & 18,4 & 160 & 81,6 \\
Desórdenes del sueño & 104 & 39,54 & 18 & 17,3 & 86 & 82,7 \\
\hline
\end{tabular}

\section{DISCUSIÓN}

La prevalencia de TTM aparece muy baja en la niñez, aumentando en la adultez y disminuyendo nuevamente hacia la vejez. Las afecciones son escasas en niños de entre 3 y 6 años. Según la literatura consultada, la mayor prevalencia de TTM se encuentra en el rango 30-45 años (12). En el presente estudio, la mayor cantidad de individuos se encontró en el rango etario de 19-29 años, lo que podría indicar que los factores predisponentes y desencadenantes, en nuestra población, aparecen a más temprana edad.
La mayor prevalencia de TTM en mujeres, fue coincidente con todos los estudios publicados. En general las mujeres sufren con más frecuencia de dolor crónico y relatan dolor con mayor intensidad y duración que los hombres (13). LeResche $(14,15)$ concluye que la asociación entre género y dolor es muy compleja, dada las diferencias existentes en las condiciones anatómicas, fisiológicas y hormonales. En general, la prevalencia de TTM, tanto de origen muscular como articular, en mujeres, sobre todo en edad fértil, es 2:1 respecto de los hombres.

\section{TABLA 4.- APROXIMACIÓN DIAGNÓSTICA DEL COMPONENTE PSICOSOCIAL}

\begin{tabular}{|c|c|c|c|c|c|c|c|}
\hline \multicolumn{8}{|c|}{ Evaluación psicosocial } \\
\hline \multirow{3}{*}{ Grado de depresión } & \multirow{2}{*}{\multicolumn{2}{|c|}{ Depresión }} & \multirow{3}{*}{ Somatización } & \multicolumn{4}{|c|}{ Signos físicos } \\
\hline & & & & \multicolumn{2}{|c|}{ con dolor } & \multicolumn{2}{|c|}{ sin dolor } \\
\hline & $\mathbf{n}$ & $\%$ & & $\mathbf{n}$ & $\%$ & $\mathbf{n}$ & $\%$ \\
\hline No & 124 & 47,15 & No & 119 & 45,25 & 163 & 61,98 \\
\hline Moderada & 81 & 30,80 & Moderada & 109 & 41,44 & 70 & 26,62 \\
\hline Severa & 57 & 21,67 & Severa & 34 & 12,93 & 28 & 10,65 \\
\hline $\mathrm{n} / \mathrm{r}$ & 1 & 0,38 & $\mathrm{n} / \mathrm{r}$ & 1 & 0,38 & 2 & 0,76 \\
\hline Total & 263 & 100 & Total & 263 & 100 & 263 & 100 \\
\hline
\end{tabular}


Huang y cols. (16) encontraron una asociación significativa entre género femenino y dolor miofascial y dolor miofascial asociado a artralgia. En este mismo sentido, un estudio de casos y controles realizado por Velly y cols. (17) concluyó que las mujeres presentaban tres veces más riesgo de presentar dolor miofascial crónico que los hombres.

Algunos estudios han sugerido que los mecanismos moduladores del dolor difieren entre hombres y mujeres (18). En un estudio realizado donde se evaluaba la respuesta frente a estimulación nociva, se concluyó que las mujeres con TTM exhibieron mayor sumación temporal de dolor; mayor disconformidad; y mayor frecuencia de postsensaciones dolorosas que los controles sanos. Lo propio ocurrió en el grupo control, respecto de los hombres. Esto sugiere diferencias en los centros superiores de regulación (19). Diversas alteraciones psicosociales como depresión, ansiedad y catastrofismo, se presentan con mayor frecuencia en mujeres y podrían contribuir en alguna medida a aumentar el riesgo de aparición de dolor (20).

El motivo de consulta más frecuente, sobre la base de un cuestionario de 9 grupos de variables, fue el dolor cráneo-cérvicofacial, lo que confirma lo encontrado en otras investigaciones, en el sentido de que es el dolor, el que con más frecuencia, determina la búsqueda de ayuda sanitaria (21). En segundo lugar aparecen las parafunciones. Esto evidenciaría un reconocimiento por parte de los enfermos, de los efectos patogénicos que ellas tendrían.

El número de pacientes distribuidos según rangos de tiempo de evolución del problema, demuestra la cronicidad de los TTM. Prácticamente un tercio de la muestra relató una evolución de entre 1-5 años. Solo un quinto refirió una condición aguda, es decir, menos de 6 meses de evolución. En todo caso, el tiempo transcurrido debe ser interpretado con precaución, ya que se reconoce que en períodos superiores a un año, el sujeto pierde precisión en sus recuerdos. Por otra parte, nos parece, que la condición de cronicidad podría interpretarse también como la ineficacia del sistema sanitario para resolver prontamente la patología.

Llama la atención, la distribución de los pacientes de acuerdo a la forma de inicio de su problema, con variables excluyentes, donde casi la mitad de los enfermos no identifica la causa de su afección. Esto podría relacionarse con la característica multifactorial de la etiología de los TTM, lo que dificultaría a la persona, establecer una relación de causalidad simple. La causa mayormente identificada es la variable definida como "estrés-depresión", en una cuarta parte de los individuos estudiados. Es interesante consignar, que menos de un décimo de los enfermos asocia el inicio del TTM a alguna acción odontológica, principalmente, exodoncia de terceros molares y terapia ortodóntica.

La frecuencia relativa de comorbilidad, con variables no excluyentes, demostró la existencia de cefaleas en más de la mitad de los individuos evaluados. Algunos estudios señalan que los TTM serían un factor de riesgo para los dolores de cabeza (22). Por otro lado, al mejorar los síntomas de los TTM, también se consigue una disminución de las cefaleas (23).

La presencia de dolor en la columna cervical también comprometió a un grupo cercano al 50\%, siendo coincidente con lo reportado por otros estudios. La prevalencia de desórdenes de la columna cervical (DCC) en pacientes con TTM varía entre un $23 \%$ a un $70 \%$ comparado con el $5 \%$ al $31 \%$ en grupos controles y pacientes con DCC reportan más signos y síntomas de TTM que controles sanos (1).

Se observó una asociación significativa entre dolor muscular local y los grados de depresión severa y moderada $\left(\chi^{2}=7,814 ; p=0,00518\right)$. Varios estudios muestran que pacientes con cuadros miogénicos presentan más dolor y distrés que aquellos con cuadros relacionados con la articulación (16). Stephen y col. (24) afirman que, si bien no es despreciable cuando se trata de un problema de origen articular, la influencia de los factores psicológicos es mayor cuando el origen del problema es muscular.

No se observó asociación significativa entre los factores oclusales definidos en el estudio y las categorías diagnósticas estudiadas. Sin embargo, bruxismo y dolor muscular local mostraron una dependencia estadísticamente significativa $(p=0,014)$, al igual que bruxismo y capsulitis/sinovitis $(p=0,0255)$. Isacsson y col. (25) encontraron que pacientes con dolor miogénico reportaron más bruxismo y disconfort dentario. 
El componente psicosocial arrojó que un 52,47\% de los sujetos presentó algún grado de depresión. Estos datos son similares a los encontrados en la literatura. Yap y cols. (12) encontraron en un grupo de pacientes asiáticos, que la depresión estaba de presente en el 39,8\% y la somatización, en el $47,6 \%$ de los individuos evaluados. Un estudio similar hecho por Plesh (21) determinó que la depresión moderada y severa estaba presente en un $28 \%$ y $36 \%$ respectivamente, en tanto que la somatización moderada con dolor se presentaba en un $50 \%$.

Desde 1950 se ha acumulado evidencia de que los factores psicológicos son de importancia en ciertos subgrupos de pacientes TTM (26). Por otro lado, estudios señalan que las alteraciones psicológicas, como la depresión, la ansiedad y el catastrofismo, podrían contribuir en alguna medida a aumentar el riesgo de aparición de dolor en los individuos (20). Depresión y somatización han mostrado influenciar la expresión de signos y síntomas de los TTM, por lo tanto, al igual que en los otros casos de comorbilidad, el componente psicosocial también determina un enfoque terapéutico multidisciplinario.

Disturbios del sueño estuvieron presentes en un $39,54 \%$ de los pacientes. Esta condición es frecuente en enfermos con TTM, señalándose que habría una relación con el dolor y las alteraciones psicosociales. Selaimen y cols. (27), señalan que el sueño y la depresión son considerados importantes indicadores de riesgo para el desarrollo de los TTM.

Dada la alta prevalencia de malos hábitos, y la asociación estadística significativa entre bruxismo y dolor muscular local, y entre capsulitis sinovitis y bruxismo, la terapéutica debe incluir mecanismos de autorregulación física e intercepción de parafunciones; además de la colaboración interdisciplinaria en el manejo de los enfermos.

En lo sucesivo se plantea aumentar el número de pacientes de la base de datos.

\section{BIBLIOGRAFÍA}

1. de Leeuw R, editor. Orofacial pain guidelines for assessment, diagnosis and management. $4^{\circ}$ Edition. Quintessence Books; 2008.
2. Seligman DA, Pullinger AG. The Role of Intercuspal Occlusal Relationships in Temporomandibular Disorders: A Review. J Craniomandib Disord 1991;5(2):96-106.

3. McNamara JA Jr, Seligman DA, Okeson JP. Occlusion, Orthodontic treatment, and temporomandibular disorders: a review. J Orofac Pain 1995;9(1):73-90.

4. Gesch D, Bernhardt O, Kirbschus A. Association of malocclusion and functional occlusion with temporomandibular disorders (TMD) in adults: a systematic review of population-based studies. Quintessence International 2004;35(3):211-221.

5. Landi $N$, Manfredini D, Tognini F, Romagnoli $M$, Bosco M. Quantification of the relative risk of multiple occlusal variables for muscle disorders of the stomatognathic system. The Journal of Prosthetic Dentistry 2004;92(2):190-5.

6. Koh H, Robinson KH. Occlusal adjustment for treating and preventing temporomandibular joint disorders. J Oral Rehabil 2004;31(4):287-92.

7. Okeson JP. Diagnóstico de los trastornos témporomandibulares. En Tratamiento de oclusión y afecciones témporomandibulares. Mosby: Madrid;2003.

8. Dworkin SF, LeResche L. Research diagnostic criteria for temporomandibular disorders: review, criteria, examinations and specifications, critique. J Craniomandib Disord. 1992;6(4):301-55.

9. Buysse DJ, Reynolds III ChF, Monk TH., Berman SR, Kupfer DJ. The Pittsburgh Sleep Quality Index: A New Instrument for Psychiatric Practice and Research. Psychiatry Research 1989;28:193213.

10. Beighton PH, Horan FT : Orthopaedic aspects of the Ehlers-Danlos Syndrome. J Bone Joint Surg 1969;51B:114-49.

11. Pullinger AG, Seligman DA, Gornbein JA: A multiple logistic regression analysis of the risk and relative odds of temporomandibular disorders as 
a function of common occlusal features. J Dent Res 1993;72:968-979.

12. Yap AU, Dworkin SF, Chua EK, List T, Tan KB, Tan HH. Prevalence of temporomandibular disorder subtypes, psychologic distress, and psychosocial dysfunction in Asian patients. J Orofac Pain 2003;17:21-8.

13. Unruh AM. Gender variations in clinical pain experience. Pain 1996;65(23):123-67.

14. LeResche L, Saunders K, Von Korff MR, Barloes W, Dworkin SF. Use of exogenous hormones and risk of temporomandibular disorder pain. Pain 1997;69:153-60.

15. LeResche L. Epidemiology of temporomandibular disorders: implications for the investigation of etiologic factors. Crit Rev Oral Biol Med 1997;8: 291-305.

16. Huang GJ, LeResche L, Critchlow CW, Martin MI, Drangsholt MT. Risk factors for diagnostic subgroups of painful temporomandibular disorders (TMD). J Dent Res 2002; 81:284-8.

17. Velly AM, Gornitsky M, Philippe P. Contributing factors to chronic myofascial pain: a case-control study. Pain 2003;104:491-9.

18. Bereiter DA. Sex differences in brainstem neural activation after injury to the TMJ region. Cells Tissues Organs 2001;169(3):226-37.

19. Sarlani E, Greenspan JD. Why look in the brain for answers to temporomandibular disorder pain? Cells Tissues Organs 2005;180:69-75.

20. Weissman MM., Olfson M. Depression in women: implications for health care research. Science 1995;269:799-801.

21. Plesh O, Sinisi SE, Crawford PB, Gansky SA. Diagnoses based on the Research Diagnostic Criteria for Temporomandibular Disorders in a biracial population of young women. J Orofac Pain 2005; 19:65-75.

22. Bernhardt O, Gesch D, Schwahn C, Mack F, Meyer $\mathrm{G}$, John $U$, et al. Risk factors for headache, including TMD signs and symptoms, and their impact on quality of life. Results of the Study of Health in Pomerania (SHIP). Quintessence Int 2005;36(1):55-64.

23. Ekberg EC, Nilner M. Treatment outcome of short- and long-term appliance therapy in patients with TMD. J Oral Rehabil 2006;33(10):713-21.

24. Stephen M, Auerbach D, Laskin D, Frantsve M, Orr T. Depression, pain, exposure to stressful life events, and long-term outcomes in temporomandibular disorder patients. Journal of Oral and Maxillofacial Surgery 2001;59(6): 628-33.

25. Isacsson G, Linde C, Isberg A. Subjective symptoms in patients with temporomandibular joint disk displacement versus patients with myogenic craniomandibular disorders. J Prosthet Den 1989t;61:70-7.

26. Sipilä K, Ylöstalo PV, Joukamaa M, Knuuttila M. Comorbidity between facial pain, widespread pain and depressive symptoms in young adults. J Orofac Pain 2006;20(1):24-30.

27. Selaimen CM, Jeronymo JC, Brilhante Dl, Grossi ML. Sleep and depression as risk indicators for temporomandibular disorders in a cross-cultural perspective: a case-control study. Int J Prosthodont 2006;19:154-61.

\section{CORRESPONDENCIA}

Walther Meeder Bella

Facultad de Odontología. Universidad de Valparaíso Subida Carvallo 221 Playa Ancha, Valparaíso Chile.

Correo electrónico: wmeeder@uv.cl 